\title{
ON THE REPRESENTATION OF THE SYMMETRICAL NUCLEUS OF A LINEAR INTEGRAL EQUATION
}

\author{
$B y$ E. W. Hobson.
}

[Received February 10th, 1914.--Read February 12th, 1914.]

IF $K(s, t)$ denote a symmetrical function of $s, t$ defined in the fundamental square $a \leqslant s \leqslant b, a \leqslant t \leqslant b$, then it may be regarded as the nucleus (Kern) of the linear integral equation

$$
f(s)=\phi(s)-\lambda \int_{a}^{b} K(s, t) \phi(t) d t .
$$

If $\left\{\phi_{n}(s)\right\},\left\{\lambda_{n}\right\}$ denote the characteristic functions and numbers of the equation corresponding to the nucleus $K(s, t)$, the following fundamental theorems relating to the representation of $K(s, t)$ are well known for the case in which $K(s, t)$ is a continuous function :-

(a) If the series $\sum_{n=1} \frac{\phi_{n}(s) \phi_{n}(t)}{\lambda_{n}}$ converges uniformly in the fundamental square, then $K(s, t)$ is its sum-function.

(b) If all the characteristic numbers $\left\{\lambda_{n}\right\}$ with the possible exception of a finite number of them are of one and the same sign, then $\sum_{n=1} \frac{\phi_{n}(s) \phi_{n}(t)}{\lambda_{n}}$ certainly converges uniformly to the sum $K(s, t)$. This theorem was first established by Mercer.

It can easily be shewn that the uniform convergence of the series in the whole square necessarily involves its absolute convergence at each point.

The present communication contains the results obtained in the course of an endeavour to ascertain how far the nucleus $K(s, t)$ is represented by the series when that nucleus is not necessarily continuous, but may have finite or infinite discontinuities in the fundamental square. In many of the most important applications of the theory of linear integral 
equations the crse in which $K(s, t)$ is discontinuous on the line $s=t$ arises; it is therefore desirable on this account, as well as on the general ground of the desirability of restricting the generality of the theorems as little as possible, that the relation of the series to a discontinuous nucleus should be elucidated. Throughout the investigation it is assumed that all the integrals employed exist in accordance with Lebesgue's definition of an integral. The Riesz-Fischer theory relating to series of normal funetions and to integral equations of the first kind is extensively used.

It is shown that a nucleus $K(s, t)$ such that $\int_{a}^{b}\left\{\left.K(s t)\right|^{2} d t\right.$ is a limited function of $s$ exists, such as to have prescribed characteristic functions and numbers $\left\{\phi_{n}(s)\right\},\left\{\lambda_{n}\right\}$, provided the series $\sum_{n=1}\left\{\frac{\phi_{n}(s)}{\lambda_{n}}\right\}^{2}$ converges for each value of $s$ to a value which is a limited function of $s$ in $(a, b)$. It is further shewn that the function $K(s, t)$ determined in accordance with the prescribed conditions is unique, except for equivalent functions not differing essentially from it. Several theorems are deduced from this result, and a simple proof of Mercer's theorem is obtained by these means.

By means of an extension of a well known theorem due to Hilbert, to the case of a discontinuous nucleus, an extension of Mercer's theorem is obtained which applies to all nuclei which are not intinitely discontinuous on the line $s=t$. Various other special theorems are obtained relating to the convergence of the series.

It is shown that, in case the repeated function of $K(s, t)$ is continuous, or at least equivalent to a continuous function, the nucleus $K(s, \mathrm{t})$ may be divided into the sum of two functions $K^{(1)}(s, t), K^{(2)}(s, t)$ that are orthogonal to one another and are such that $K^{(1)}(s, t)$ has for its sole characteristic numbers those characteristic numbers of $K(s, t)$ that are positive, and that $K^{(2)}(s, t)$ has for its sole characteristic numbers those characteristic numbers of $K(s, t)$ that are negative. The characteristic function corresponding to a characteristic number of $K^{(1)}(s, t)$ or of $K^{(2)}(s, t)$ is the same as the characteristic number of $K(s, t)$ corresponding to the same characteristic number. This result may possibly help to decide whether, or under what conditions, Mercer's theorem can be extended to the case of a continuous nucleus that has an infinite number of characteristic numbers of each sign. The question is shewn to depend upon whether $K^{(1)}(s, t)$ and $K^{(2)}(s, t)$ are infinitely discontinuous, or not, upon the line $s=t$.

1. If $K(s, t)$ be a summable and symmetrical function defined for the points of the square $a \leqslant s \leqslant b, a \leqslant t \leqslant b$, it may be regarded as the 
nucleus of the linear integral equation

$$
f(s)=\phi(s)-\lambda \int_{a}^{b} K(s, t) \phi(t) d t,
$$

with symmetrical nucleus.

The method of Fredholm is directly applicable to the equation in case $K(s, t)$ is a limited function. In case $K(s, t)$ is not limited, but when all the repeated nuclei from and after the one of some fixed order are limited, Fredholm's method is capable of extension so as to provide the solution of the integral equation.

If $K_{n}(s, t), K_{n+1}(s, t), \ldots$, are all limited, and $\phi_{r}(s)$ is a characteristic function for the nucleus $K_{n}(s, t)$, with $\lambda_{r}^{n}$ as the corresponding characteristic number, then $\phi_{r}(s)$ is also, ${ }^{*}$ in general, a characteristic function for the nucleus $K(s, t)$, the corresponding characteristic number being $\lambda_{r}$, a real $n$-th root of $\lambda_{r}^{n}$; and, conversely, every characteristic function for $K(s, t)$ with the corresponding charncteristic number can be obtained in this manner. The case in which $n$ is even, and two or more characteristic functions correspond to one and the same characteristic number, is exceptional.

Thus, let

$$
\frac{\phi_{r}^{(1)}(s) \phi_{r}^{(1)}(t)+\phi_{r}^{(2)}(s) \phi_{r}^{(2)}(t)}{\lambda^{n}-\lambda_{r}^{n}}
$$

be that part of the resolvant for the nucleus $K_{n}(s, t)$ which becomes infinite when $\lambda^{n}=\lambda_{r}^{n}$. In this case the characteristic functions $\phi_{r}^{(1)}(s)$, $\phi_{r}^{(2)}(s)$ are not uniquely determinate. For we may replace $\phi_{r}^{(1)}(s), \phi_{r}^{(2)}(s)$ by $\cos \alpha \cdot \phi_{r}^{(1)}(s)+\sin \alpha \cdot \phi_{r}^{(2)}(s), \sin \alpha \cdot \phi_{r}^{(1)}(s)-\cos \alpha \cdot \phi_{r}^{(2)}(s)$ respectively, where $\alpha$ is arbitrary, without altering the expression $\phi_{r}^{(1)}(s) \phi_{r}^{(1)}(t)+\phi_{r}^{(2)}(s) \phi_{r}^{(2)}(t)$. The corresponding part of the resolvant of $K(s, t)$ will bet of one of the forms

$$
\begin{gathered}
\frac{\phi_{r}^{(1)}(s) \phi_{r}^{(1)}(t)+\phi_{r}^{(2)}(s) \phi_{r}^{(2)}(t)}{\lambda-\lambda_{r}}, \\
\frac{\phi_{r}^{(1)}(s) \phi_{r}^{(1)}(t)+\phi_{r}^{(2)}(s) \phi_{r}^{(2)}(t)}{\lambda+\lambda_{r}}, \\
\frac{\left[\cos \alpha \cdot \phi_{r}^{(1)}(s)+\sin \alpha \cdot \phi_{r}^{(2)}(s)\right]\left[\cos \alpha \cdot \phi_{r}^{(1)}(t)+\sin \alpha \cdot \phi_{r}^{(2)}(t)\right]}{\lambda-\lambda_{r}} \\
+\frac{\left[\sin \alpha \cdot \phi_{r}^{(1)}(s)-\cos \alpha \cdot \phi_{r}^{(1)}(s)\right]\left[\sin \alpha \cdot \phi_{r}^{(1)}(t)-\cos \alpha \cdot \phi_{r}^{(2)}(t)\right]}{\lambda+\lambda_{r}},
\end{gathered}
$$

* See my paper "On the Linear Integral Equation," Proc. London Math. Soc., Ser. 2, Vol. 13, p. 307 .

$\dagger$ Ibid., p. 332, where it is shewn that there is one canonical sub-group for $\bar{K}(s, t, \lambda)$ corresponding to each such sub-group for $\bar{K}_{\mu \prime}\left(s, t, \lambda^{\prime \prime}\right)$, and vice versa. 
where $\alpha$ has some particular value. It is now clear that the characteristic functions for $K_{n}(s, t)$, corresponding to $\lambda^{n}=\lambda_{r}^{n}$, may be so chosen that they are also characteristic functions for the nucleus $K(s, t)$ corresponding to $\lambda=\lambda_{r}$, to $\lambda=-\lambda_{r}$, or to the two characteristic numbers $\lambda=\lambda_{r}$, $\lambda=-\lambda_{r}$.

We may thus in all cases regard the characteristic functions for $K(s, t)$ as identical with those for $K_{n}(s, t)$.

Unless $K(s, t)$ is in some way restricted it will not necessarily be the case that the characteristic functions $\left\{\phi_{r}(s)\right\}$ are continuous functions.

It is, however, a sufficient condition that they may all be continuous, that $K(s, t)$ should be a limited function with its discontinuities regularly distributed, in accordance with the extended meaning of that term given in the paper referred to above. It is there shewn that, if $\phi(t)$ is any summable function, then for such a function $K(s, t), \int_{a}^{b} K(s, t) \phi(t) d t$ is a continuous function of $s$; thus the relation

$$
\phi_{r}(s)=\lambda_{r} \int_{a}^{b} K(s, t) \phi_{r}(t) d t
$$

can be satisfied only by a continuous function $\phi_{r}(s)$.

If $K(s, t)$ be unlimited, but $K_{n}(s, t), K_{n+1}(s, t), \ldots$, be limited functions, it is sufficient to ensure the continuity of the characteristic functions that $K_{n}(s, t)$ should have its discontinuities regularly dis tributed, in the sense referred to above.

When $K(s, t)$ is any symmetrical summable function that differs from zero at points of a set of which the plane measure is greater than zero, the proof due to Kneser* that $K(s, t)$ has at least one characteristic number is applicable, whether the function be continuous or not, provided it be limited. In case the function is unlimited, but the repeated nuclei $K_{n}(s, t), K_{n+1}(s, t), \ldots$, are limited, the proof establishes that $K_{*}(s, t)$ has at least one characteristic number, and this will also be a characteristic number for $K(s, t)$.

'I'wo symmetrical nuclei $K(s, t), K^{\prime}(s, t)$ of the linear integral equation

$$
f(s)=\phi(s)-\lambda \int_{a}^{b} K(s, t) \phi(t) d t
$$

will be said to be equivalent to one another when they differ from one another only at points of some set such that its section by any straight line parallel to either the $s$ - or $t$-axis is a set of which the linear measure

* See the Rendiconti di Palermo, Vol. xxı, p. 235. 
is zero. It is clear that equivalent nuclei have the same characteristic functions $\left\{\phi_{n}(s)\right\}$ and the same characteristic constants $\left\{\lambda_{n}\right\}$. Also they have the same repeated nuclei.

2. Let it be assumed that the symmetrical nucleus $K(s, t)$, although not necessarily continuous or limited, is such that $\int_{a}^{b}\{K(s, t)\}^{2} d s$ is a limited function of $t$, and that the repeated nucleus $\int_{a}^{b} K\left(s, t^{\prime}\right) K\left(t, t^{\prime}\right) d t^{\prime}$ is either equal to, or equivalent to, the continuous function $K_{2}(s, t)$. The characteristic functions $\left\{\phi_{n}(s)\right\}$ are then all continuous.

It will then be shewn that $\sum_{n=1} \frac{\phi_{n}(s) \phi(t)}{\lambda_{n}^{2}}$ converges uniformly to the value of $K_{2}(s, t)$; the convergence being absolute.

Since

$$
\int_{a}^{b}\left\{K(s, t)-\sum_{n=1}^{n=m} \frac{\phi_{n}(s) \phi_{n}(t)}{\lambda_{n}}\right\}^{2} d s=\int_{a}^{b}\{K(s, t)\}^{2} d s-\sum_{n=1}^{n=m} \frac{\left\{\phi_{n}(t)\right\}^{2}}{\lambda_{n}^{2}},
$$

it follows that the series $\sum_{n=1} \frac{\left\{\phi_{n}(t)\right\}^{2}}{\lambda_{n}^{2}}$ is convergent for every value of $t$, and that its sum is $\leqslant \int_{a}^{b}\{K(s, t)\}^{2} d s$, which is less, for every value of $t$, than some fixed positive number $M$. We have

$$
\left\{\sum_{n=m}^{n+r}\left|\frac{\phi_{n}(s) \phi_{n}(t)}{\lambda_{n}^{2}}\right|\right\}^{2} \leqslant \sum_{n=m}^{m+r} \frac{\left\{\phi_{n}(s)\right\}^{2}}{\lambda_{n}^{2}} \sum_{n=m}^{m+r} \frac{\left\{\phi_{n}(t)\right\}^{2}}{\lambda_{n}^{2}}<M \sum_{n=m}^{m+r} \frac{\left\{\phi_{n}(t)\right\}^{2}}{\lambda_{n}^{2}} .
$$

For a fixed value of $t$, let $m$. be so chosen that

$$
\sum_{n=m}^{m+r} \frac{\left\{\phi_{n}(t)\right\}^{2}}{\lambda_{n}^{2}}<\frac{\epsilon^{2}}{M},
$$

for every value of $r$, where $\epsilon$ is an arbitrarily chosen positive number; we have then

$$
\left|\sum_{n=m}^{m+r} \frac{\phi_{n}(s) \phi_{n}(t)}{\lambda_{n}^{2}}\right| \leqslant \sum_{n=m}^{m+r}\left|\frac{\phi_{n}(s) \phi_{n}(t)}{\lambda_{n}^{2}}\right|<\epsilon
$$

for every value of $r$. Since $e$ is arbitrary, it follows that, for a fixed value of $t$, the series $\sum_{n=1} \frac{\phi_{n}(s) \phi_{n}(t)}{\lambda_{n}^{2}}$ converges absolutely and uniformly, and has therefore a sum that is continuous with respect to $s$. From the symmetry of the variables $s$ and $t$, we see that for all values of $s$ and $t$ the series converges to a function that is continuous with respect to the variables $s$ and $t$ separately, at every point $(s, t)$. 
Considering the series $\sum_{n=1} \frac{\left\{\phi_{n}(t)\right\}^{2}}{\lambda_{n}^{4}}$, we have

$$
\sum_{n=m}^{m+r} \frac{\left\{\phi_{n}(t)\right\}^{2}}{\lambda_{n}^{4}}<\frac{1}{\lambda_{m}^{2}} \sum_{n=m}^{m+r} \frac{\left\{\phi_{n}(t)\right\}^{2}}{\lambda_{n}^{2}}<\frac{M}{\lambda_{m}^{2}}
$$

hence, if $m$ be sufficiently large, $\sum_{n=m}^{m+r} \frac{\left\{\phi_{n}(t)\right\}^{2}}{\lambda_{n}^{4}}$ is less than an arbitrarily chosen positive number, for all values of $r$ and of $t$. Thus the series $\sum_{n=1} \frac{\left\{\phi_{n}(t)\right\}^{2}}{\lambda_{n}^{4}}$ is uniformly convergent. From this we deduce at once that the series $\sum_{n=1} \frac{\phi_{n}(s) \phi_{n}(t)}{\lambda_{n}^{4}}$ is absolutely and uniformly convergent, since

$$
\left|\phi_{n}(s) \phi_{n}(t)\right| \leqslant \frac{1}{2}\left\{\phi_{n}(s)\right\}^{2}+\frac{1}{2}\left\{\phi_{n}(t)\right\}^{2} \text {. }
$$

It follows, from a known theorem, that the series converges to $K_{4}(s, t)$; and we have

$$
K_{4}(t, t)=\sum_{n=1}^{\infty} \frac{\left\{\phi_{n}(t)\right\}^{2}}{\lambda_{n}^{4}} .
$$

Since

$$
\int_{a}^{b}\left\{K_{2}(s, t)-\sum_{n=1}^{n} \frac{\phi_{n}(s) \phi_{n}(t)}{\lambda_{n}^{2}}\right\}^{2} d s=K_{4}(t, t)-\sum_{n=1}^{n} \frac{\left\{\phi_{n}(t)\right\}^{2}}{\lambda_{n}^{4}}
$$

we now see that

$$
\lim _{m \sim \infty} \int_{a}^{b}\left\{K_{2}(s, t)-\sum_{n=1}^{m} \frac{\phi_{n}(s) \phi_{n}(t)}{\lambda_{n}^{2}}\right\}^{2} d s=0 .
$$

From this, we infer, by employing the Riesz-Fischer theory, * that for any fixed value of $t$, there exists a sequence of values of $m$, such that $\sum_{n=1}^{m} \frac{\phi_{n}(s) \phi_{n}(t)}{\lambda_{n}^{2}}$ converges to $K_{2}(s, t)$ as $m$ passes through the values of the sequence, the convergence taking place for all values of $s$ with the possible exception of those belonging to some set of linear measure zero. Since it has been shewn that the series $\sum_{n=1} \frac{\phi_{n}(s) \phi_{n}(t)}{\lambda_{n}^{2}}$ is everywhere convergent, it now follows that, for ench fixed value of $t$, it converges to $K_{2}(s, t)$, for all values of $s$ except possibly those belonging to some set of linear measure zero. The points of convergence to $K_{2}(s, t)$ are, for each value of $t$, everywhere dense on the line $a \leqslant s \leqslant b$, and the sum of the series is continuous

* See, for example, the account of this theory given by W. H. Young and G. Chisholm Young in the Quarteriy Joumal of Mathematics, Vol. XLIV, p. 47. 
with regard to $s$; therefore the series converges to the continuous function $K_{2}(s, t)$ for every value of $s ; t$ having any fixed value.

It has now been shewn that $\sum_{n=1} \frac{\phi_{n}(s) \phi_{n}(t)}{\lambda_{n}^{2}}$ converges for all values of $s$ and $t$ to the sum $K_{3}(s, t)$.

Since $\sum_{n=1} \frac{\left\{\phi_{n}(s)\right\}^{2}}{\lambda_{n}^{2}}$, in which all the terms are positive, converges to the continuous function $K_{2}(s, s)$, its convergence must be uniform.

Since

$$
\left|\sum_{n=m}^{m+r} \frac{\phi_{n}(s) \phi_{n}(t)}{\lambda_{n}^{2}}\right| \leqslant \sum_{n=m}^{m+r} \frac{\left.\mid \phi_{n}(s) \phi_{n}(t)\right\}}{\lambda_{n}^{2}}<\frac{1}{2} \sum_{n=m}^{m+r} \frac{\left\{\phi_{n}(s)\right\}^{2}}{\lambda_{n}^{2}}+\frac{7}{2} \sum_{n=m}^{m+r} \frac{\left\{\phi_{n}(t)\right\}^{2}}{\lambda_{n}^{2}}
$$

the integer $m$ can be so chosen that, for all values of $r$,

$$
\sum_{n=m}^{m+r} \frac{\left|\phi_{n}(s) \phi_{n}(t)\right|}{\lambda_{n}^{2}} \text { and }\left|\sum_{n=m}^{m+r} \frac{\phi_{n}(s) \phi_{n}(t)}{\lambda_{n}^{2}}\right| \text {, }
$$

are less than an arbitrarily chosen number, for all values of $(s, t)$. It follows that the convergence of $\sum_{n=1} \frac{\phi_{n}(s) \phi_{n}(t)}{\lambda_{n}^{2}}$ to $K_{2}(s, t)$ is uniform and
absolute.

The following theorem has been established:-

I. If $K(s, t)$ is any symmetrical nucleus, whether continuous or not, and for which $\int_{a}^{b}\{K(s, t)\}^{2} d s$ is less than some fixed positive number independent of $t$, and if $K(s, t)$ is such that its repeated nucleus is equal to, or equivalent to, a continuous function $K_{2}(s, t)$, then $\sum_{n=1} \frac{\phi_{n}(s) \phi_{n}(t)}{\lambda_{n}^{2}}$ connerges uniformly and absolutely to the value of $K_{2}(s, t)$.

The case of this theorem in which $K(s, t)$ is continuous has been established by Kowalewski.*

3. It is known that, if $\phi_{1}(t), \phi_{2}(t), \ldots$, be a sequence of orthogonal functions defined for the interval $(a, b)$, and normalized so that

$$
\int_{a}^{b}\left\{\phi_{n}(t)\right\}^{2} d t=1
$$

and if $c_{1}, c_{2}, \ldots, c_{n}, \ldots$, be a sequence of real numbers, the necessary and sufficient condition that a function $F(t)$, of which the square is summable

* See his treatise Einfilhrung in die Determinantentheorie. 
in $(a, b)$, should exist and be such as to satisfy the conditions

$$
\int_{a}^{b} F(t) \phi_{n}(t) d t=c_{n}, \quad \text { for } \quad n=1,2,3, \ldots
$$

is that the series $c_{1}^{2}+c_{2}^{2}+\ldots+c_{n}^{2}+\ldots$ should be convergent.

The function $F(t)$ is unique, disregarding the distinction between functions that differ from one another at points of a set of measure zero, only in case the set of orthogonal functions is closed; i.e. when no other function orthogonal to them all exists, such that its square is summable, that it differs from zero at points of a set of measure greater than zero, and such that it is not a linear function of a finite number of the given orthogonal functions. Even when the set of orthogonal functions is not closed the function $F(t)$ can be so determined that

$$
\int_{a}^{b}\{F(t)\}^{2} d t
$$

is equal to the sum of the series $c_{1}^{2}+c_{2}^{2}+\ldots$.

If $\chi(t)$ is any independent function, of which the square is summable, and such as to be orthogonal to all the functions $\left\{\phi_{n}(t)\right\}$, then we have

$$
\int_{a}^{b} F(t) \chi(t) d t=0
$$

The truth of this last statement follows from a consideration of the fact that

$$
\int_{a}^{b}\left[F(t)-\chi(t) \int_{a}^{b} F(t) \chi(t) d t-\sum_{n=1}^{m} c_{n} \phi_{n}(t)\right]^{2} d t
$$

has a value that is essentially positive. It is equivalent to

$$
\int_{a}^{b}\{F(t)\}^{2} d t-\left\{\int_{a}^{b} F(t) \chi(t) d t\right\}^{2}-\sum_{n=1}^{n} c_{n}^{2}
$$

it being assumed that $\quad \int_{a}^{b}\{\chi(t)\}^{2} d t=1$.

As this expression is positive for every value of $m$, we see that

$$
\int_{a}^{b} F(t) \chi(t) d t=0
$$

Let us now suppose that $\lambda_{1}, \lambda_{2}, \ldots, \lambda_{n}, \ldots$ is any sequence of real numbers arranged in order of increasing absolute magnitude, and such that $\left|\lambda_{n}\right|$ increases indefinitely as $n$ does so. 
Let it be assumed that, for a fixed number $s$ in the interval $(a, b)$, the series $\sum_{n=1} \frac{\left\{\phi_{n}(s)\right\}^{2}}{\lambda_{n}^{2}}$ is convergent.

Then the equations

$$
\frac{\phi_{n}(s)}{\lambda_{n}}=\int_{a}^{b} F(t) \phi_{n}(t) d t \quad(n=1,2,3, \ldots)
$$

are all satisfied by a function $F(t)$, such that

$$
\int_{a}^{b}\{F(t)\}^{2} d t=\sum_{n=1}^{\infty} \frac{\left\{\phi_{n}(s)\right\}^{2}}{\lambda_{n}^{2}}
$$

Conversely, the convergence of the series is necessary for the existence of the function $F^{\prime}(t)$, such that its square is summable and that it satisfies the equations. The function $F(t)$ is unique in the sense that it can be replaced only by another funetion which differs from it at points of a set of which the measure is zero.

When we consider different values of $s$ for which the series $\sum_{n=1} \frac{\left\{\phi_{n}(s)\right\}^{2}}{\lambda_{n}^{2}}$ is convergent, we may denote $F(t)$ by $K(s, t)$.

Let it now be assumed that $\sum_{n=1} \frac{\left\{\left.\phi_{n}(s)\right|^{2}\right.}{\lambda_{n}^{2}}$ is convergent for all values of $s$ in the interval $(a, b)$. Then $K(s, t)$ is defined, except that it may be replaced by a function which differs from it at points of a set of which the section by each line parallel to the $t$-axis has linear measure zero.

The function $K(s, t)$, so defined, is such that

$$
\phi_{n}(s)=\lambda_{n} \int_{a}^{b} K(s, t) \phi_{n}(t) d t
$$

for all values of $n$. Also, if $\chi(t)$ is any independent function such that $\chi(s)$ is orthogonal to all the functions $\left\{\phi_{n}(s)\right\}$, we have

$$
\int_{a}^{b} K(s, t) \chi(t) d t=0,
$$

and thus $\chi(t)$ is not a characteristic function.

We have now

$$
\int_{a}^{b}\left\{K(s, t)-\sum_{n=1}^{n=m} \frac{\phi_{n}(s) \phi_{n}(t)}{\lambda_{n}}\right\}^{2} d t=\int_{a}^{b}\{K(s, t)\}^{2} d t-\sum_{n=1}^{n=m} \frac{\left\{\phi_{n}(s)\right\}^{2}}{\lambda_{n}^{2}} ;
$$

and therefore for each fixed value of $s$ we have

$$
\lim _{m \sim \infty} \int_{a}^{b}\left\{K(s, t)-\sum_{n=1}^{n=m} \frac{\phi_{n}(s) \phi_{n}(t)}{\lambda_{n}}\right\}^{2} d t=0 .
$$


From this it follows, by using the Riesz-Fischer theory, that for each value of $s$ a sequence of values of $m$ can be determined so that

$$
\sum_{n=1}^{n=m} \frac{\phi_{n}(s) \phi_{n}(t)}{\lambda_{n}}
$$

converges to $K(s, t)$, except for a set of values of $t$ of measure zero, as the values of the sequence are assigned to $m$. It thus appears that the function $K(s, t)$ is symmetrical, and that it may be regarded as the nucleus of an integral equation of Fredholm's type, of which the functions $\left\{\phi_{n}(s)\right\}$ are the characteristic functions and $\left\{\lambda_{n}\right\}$ the characteristic constants.

The following theorem has now been established :-

II. If $\left\{\phi_{n}(s)\right\}$ denotes a sequence of prepared orthogonal functions for the interval $(a, b)$, and $\left\{\lambda_{n}\right\}$ denotes a sequence of real numbers in ascending order of absolute magnitude, the necessary and sufficient condition that a symmetrical nucleus $K(s, t)$ should exist, for which $\int_{a}^{b}\{K(s, t)\}^{2} d t$ is finite for each value of $s$, and for which $\left\{\phi_{n}(s)\right\}$ are the characteristic functions, and $\left\{\lambda_{n}\right\}$ the characteristic numbers, is that the series $\sum_{n=1} \frac{\left\{\phi_{n}(s)\right\}^{2}}{\lambda_{n}^{2}}$ should be convergent for all values of $s$ in $(a, b)$.

It should be observed that the plane set of values of $(s, t)$ for which no sequence of values of $m$ exists such that $\sum_{n=1}^{m} \frac{\phi_{n}(s) \phi_{n}(t)}{\lambda_{n}}$ converges to $K(s, t)$ is such that its sections by lines parallel to the axes are all sets of linear measure zero. It follows that the plane set has its plane measure zero.

In case $\sum_{n=1} \frac{\left\{\phi_{n}(s)\right\}^{2}}{\lambda_{n}^{2}}$ is divergent for a set of values of $s$ of linear measure zero, the method given fails to determine a function $K(s, t)$ for which $\left\{\phi_{n}(s)\right\}$ and $\left\{\lambda_{n}\right.$, are the characteristic functions and numbers. In this case the given functions $\left\{\phi_{n}(s)\right\}$ may be replaced by a modified set of orthogonal functions for which $K(s, t)$ may be determined so as to have the given characteristic numbers $\left\{\lambda_{n}\right\}$. Let $\bar{\phi}_{n}(s)=\phi_{n}(s)$, for all values of $s$ for which $\underset{n=1}{\Sigma} \frac{\left\{\phi_{n}(s)\right\}^{2}}{\lambda_{n}^{2}}$ is convergent, and let $\bar{\phi}_{n}(s)=0$ for all other values of $s$. The function $K(s, t)$ will then be determined, as above, for those values and $s$ and $t$ which do not belong to the exceptional set; when $s$ or $t$ belongs to the exceptional set, we assign to $K(s, t)$ the value zero. 
The equations

$$
\overline{\boldsymbol{\phi}}_{n}(s)=\lambda_{n} \int_{a}^{b} K(s, t) \overline{\boldsymbol{\phi}}_{n}(t) d t
$$

are then satisfied for all values of $s$ in $(a, b)$, and for all values of $n$.

4. Let it now be assumed that the given functions $\left\{\phi_{n}(s)\right\}$ and the given numbers $\left\{\lambda_{n}\right\}$ are such that $\sum_{n=1} \frac{\left\{\phi_{n}(s)\right\}^{2}}{\lambda_{n}^{2}}$ is not only convergent for each value of $s$, but also that its sum is a limited function of $s$, in the interval $(a, b)$.

In accordance with what has been established in $\$ 3$, a symmetrical function $K(s, t)$ exists, such that

$$
\phi_{n}(s)=\lambda_{n} \int_{a}^{b} K(s, t) \phi_{n}(t) d t, \quad \text { for } \quad n=1,2,3, \ldots,
$$

and such that

$$
\int_{a}^{b}\{K(s, t)\}^{2} d t=\sum_{n=1}^{\infty} \frac{\left\{\phi_{n}(s)\right\}^{2}}{\lambda_{n}^{2}}
$$

It will now be proved that there can exist no other function $H(s, t)$ not equivalent to $K(s, t)$, such that $\int_{a}^{b}\{H(s, t)\}^{2} d t$ is finite for each value of $s$, and limited for all values of $s$, which shall have the functions $\left\{\phi_{n}(s)\right\}$ for its sole characteristic functions, and $\left\{\lambda_{n}\right\}$ for the corresponding characteristic numbers.

For assume $H(s, t)$ to be such that

$$
\int_{a}^{b} H(s, t) \phi_{n}(t) d t=\frac{\phi_{n}(s)}{\lambda_{n}}, \text { for } \quad n=1,2,3, \ldots
$$

We need consider only the case in which $\left\{\phi_{n}(s)\right\}$ is not a closed system, for, if it were closed, $H(s, t)$ and $K(s, t)$ must be equivalent.

It is known * that any normal orthogonal system is enumerable; we

* It was shewn by E. Schmidt (Comptes Rendus de l'Academie des Sciences, Dec. 10, 1906 , p. 955), that any orthogonal set of continuous functions $\{\phi(s)\}$ must be enumerable. The proof will here be adapted by means of a slight alteration to the case of functions that are not necessarily continuous. Let $F(s)$ be any function whose square is summable in $(a, b)$, and let $\phi_{p_{1}}(s), \phi_{p_{2}}(s), \ldots, \phi_{p_{r}}(s)$ be any finite set of the functions $\{f(s)\}$; we have then

$$
\int_{a}^{b}\left\{F(s)-\sum_{l}^{r} \phi_{p_{r}}(s) \int_{a}^{b} F(s) \phi_{p_{r}}(s) d s\right\}^{2} d s=\int_{a}^{b}\{F(s)\}^{2} d s-\sum_{1}^{r}\left\{\int_{a}^{b} F^{\prime}(s) \phi_{p_{r}}(s) d s\right\}^{2} \text {. }
$$

It follows from this equality, since the expression on the right-hand side is essentially positive, that, if $k$ be any fixed positive number, there can exist only a finite number of the functions 
may therefore assume that $\left\{\phi_{n}(s)\right\}$ is a part of some* closed system of normal ortbogonal functions which is obtained by adjoining an orthogonal system $\left\{\chi_{n}(s)\right\}$, where $n=1,2,3, \ldots$, so that $\left\{\phi_{n}(s)\right\},\left\{\chi_{n}(s)\right\}$ taken together form a closed orthogonal system.

Let us suppose that

$$
\int_{a}^{b} H(s, t) \chi_{n}(t) d t=\psi_{n}(s),
$$

where $\psi_{n}(s)$ may be zero for some values of $n$, but not for all values of $n$, in which latter case $H(s, t)$ would be necessarily equivalent to $K(s, t)$.

We have now

$$
\int_{a}^{b}\{H(s, t)\}^{2} d t=\sum_{n=1}^{\infty}\left\{\psi_{n}(s)\right\}^{2}+\sum_{n=1}^{\infty} \frac{\left\{\phi_{n}(s)\right\}^{2}}{\lambda_{n}^{2}},
$$

the convergence of the series being necessary for the existence of $H(s, t)$.

$\phi(s)$, such that

$$
\left|\int_{a}^{b} F^{\prime}(s) \phi(s) d s\right|>h .
$$

Assigning to $k$ a sequence of values diminishing towards the limit zero, we see that the set of those functions $\phi(s)$, for which

$$
\left|\int_{a}^{b} F(s) \phi(s) d s\right|>0
$$

is enumerable. We have then only to consider those functions $\phi(s)$ for which

$$
\int_{a}^{b} F(s) \phi(s) d s=0 .
$$

Taking for simplicity $a=-\pi, b=\pi$, which involves no loss of generality, let $F(s)$ have successively the values $1, \cos s, \sin s, \cos 2 s, \sin 2 s, \ldots$; we have then an cnumerable set of enumerable sets in each of which one at least of the integrals is in absolute value $>0$; the whole composite set is enumerable. There is no function $\chi(s)$ such that $\int_{-\pi}^{\pi}\{x(s)\}^{2} d s$ exists as a positive number, and such that

$$
\int_{-\pi}^{\pi} \chi(s) \sin _{\sin }^{\cos } n s d s=0
$$

for every value of $n$. For, in accordance with a known theorem, first established by Fatou, for such a function $\chi(s)$ we have

$$
\frac{1}{\pi} \int_{-\pi}^{\pi}\{x(s)\}^{2} d s=\sum_{n=0}^{\infty}\left\{\int_{-\pi}^{\pi} \chi(s) \cos n s d s\right\}^{2}+\sum_{n=1}^{\infty}\left\{\int_{-\pi}^{\pi} \chi(s) \sin n s d s\right\}^{2},
$$

and thus it is impossible that all the integrals on the right-hand side can vanish. It has thus been shewn that the set $\{\phi(s)\}$ must be enumerable, whether it be closed or not.

* It may be noticed that a set of orthogonal functions may form part of more than one closed set of orthogonal functions. For example, in the interval $(-\pi, \pi)$ consider the orthogonal set $\sin x, \sin 2 x, \ldots, \sin n x, \ldots$. This forms part of the closed set $1, \cos x, \sin x$, $\cos 2 x, \sin 2 x, \ldots$; but we might also adjoin to the set $\sin x, \sin 2 x, \ldots, \sin n x, \ldots$ the orthogonal functions $P_{2}(\pi x), P_{4}(\pi x), \ldots, P_{2}(\pi x), \ldots$; where $P_{2 n}(x)$ depotes the Legendre's function of order $2 n$; the extended set is then still an orthogonal set. 
1914.] The gymmetrical nUCLels of a linear integral gquation.

Let us consider $H(s, t)-K(s, t)$ as a nucleus; we have

$$
\int_{a}^{b}\{H(s, t)-K(s, t)\} \phi_{n}(t) d t=0,
$$

and thus the functions $\left\{\phi_{n}(s)\right\}$ are not characteristic functions for this nucleus. Also

$$
\int_{a}^{b}\{H(s, t)-K(s, t)\} \chi_{n}(t) d t=\psi_{n}(s) ;
$$

and therefore it appears from these two sets of equations that

$$
\int_{a}^{b}\{H(s, t)-K(s, t)\}^{2} d t=\sum_{n=1}^{\infty}\left\{\psi_{n}(s)\right\}^{2} .
$$

It is now seen that the repeated nucleus of $H(s, t)-K(s, t)$, viz.,

$$
\int_{a}^{b}\left\{H\left(s, t^{\prime}\right)-K\left(s, t^{\prime}\right)\right\}\left\{H\left(t, t^{\prime}\right)-K\left(t, t^{\prime}\right)\right\} d t^{\prime},
$$

is numerically less than

$$
\left[\sum_{n=1}^{\infty}\left\{\psi_{n}(s)\right\}^{2} \sum_{n=1}^{\infty}\left\{\psi_{n}(t)\right\}^{2}\right]^{2}
$$

and this is a limited function since it has been assumed that

$$
\int_{a}^{b}\{H(s, t)\}^{2} d t
$$

and consequently $\sum_{n=1}^{\infty}\left\{\psi_{n}(s)\right\}^{2}$, is a limited function of $s$. It now follows, in accordance with what has been pointed out in $\$ 1$, that the nucleus $H(s, t)-K(s, t)$ has at least one characteristic function $\mu(s)$, and we have seen that this cannot belong to the set $\left\{\phi_{n}(s)\right\}$. We have therefore

$$
\int_{a}^{b}\{H(s, t)-K(s, t)\} \mu(t) d t=\frac{\mu(s)}{\nu},
$$

where $\nu$ is the characteristic number corresponding to $\mu(s)$.

From this we see that

$$
\frac{1}{\nu} \int_{a}^{b} \mu(s) \phi_{n}(s) d s=\int_{a}^{b} \mu(t) d t \int_{a}^{b}[H(s, t)-K(s, t)] \phi_{n}(s) d s=0 ;
$$

hence $\mu(s)$ is orthogonal to all the functions $\left\{\phi_{n}(s)\right\}$, and hence we have

$$
\int_{u}^{b} K(s, t) \mu(t) d t=0 .
$$

SER. 2. VOL. 14. NO. 1222 . 
It follows that

$$
\int_{a}^{b} H(s, t) \mu(t) d t=\frac{\mu(s)}{\nu} ;
$$

and therefore $\mu(s)$ is a characteristic function for the nucleus $H(s, t)$. This is contrary to the supposition that $H(s, t)$ has $\left\{\phi_{n}(s)\right\}$ for its sole characteristic functions. Hence $H(s, t)$ must be equivalent to $K(s, t)$.

The following theorem has now been established :--

III. If $\left\{\phi_{n}(s)\right\}$ be a system of normal orthogonal functions, and $\left\{\lambda_{n}\right\}$ a set of numbers arranged in order of increasing absolute magnitude, the necessary and sufficient condition that a symmetrical nucleus $K(s, t)$, such that $\int_{a}^{b}\{K(s, t)\}^{2} d t$ is a limited function of $s$, may exist and be such that $\left\{\phi_{n}(s)\right\},\left\{\lambda_{n}\right\}$ are its sole characteristic functions and numbers, is that $\sum_{n=1}\left\{\frac{\phi_{n}(s)}{\lambda_{n}}\right\}^{2}$ should converge for each value of $s$ to a value which is a limited function of $s$. This function $K(s, t)$ is the only nucleus, apart from equivalent ones, which satisfies the above conditions.

The set of values of $(s, t)$ for which no sequence of values of $m$ exists

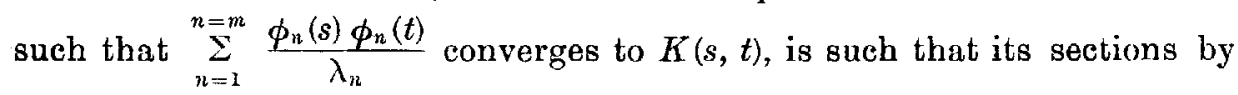
lines parallel to the axes are all of linear measure zero, and therefore the set has plane measure zero.

We have now established the following theorem:-

IV. If $K(s, t)$ is any symmetrical nucleus, not necessarily continuous or limited, but such that $\int_{a}^{b}\{K(s, t)\}^{2} d t$ exists as a limited function of $s$, for all values of $s$ in $(a, b)$, then for each set of values of $(s, t)$ with the possible exception of those belonging to a set of which all the sections by lines parallel to the axes have linear measure zero, there exists a sequence of values of $m$ such that $\sum_{n=1}^{n=m} \frac{\phi_{n}(s) \phi_{n}(t)}{\lambda_{n}}$ converges to $K(s, t)$ as $m$ passes through the values in the sequence.

If, at any point $(s, t)$ not belonging to the exceptional set of points, the series $\sum_{n=1} \frac{\phi_{n}(s) \phi_{n}(t)}{\lambda_{n}}$ is convergent, it must converge to the value $K(s, t)$; we have then the following theorem :-

V. If the series $\sum_{n=1} \frac{\phi_{n}(s) \phi_{n}(t)}{\lambda_{n}}$ corresponding to a nucleus $K(s, t)$, for 
which $\int_{a}^{b}\{K(s, t)\}^{2} d t$ is a limited function of $s$, be such that it is convergent for every point $(s, t)$ which does not belong to some set of plane measure zero, then the series converges to $K(s, t)$ with a similar exception. If the series is everywhere convergent, it converges to $K(s, t)$ except possibly at points of a set of which the sections by lines parallel to the axes have linear measure zero.

The following theorem is immediately deducible:-

VI. If $K(s, t)$ be such that the functions $\phi_{n}(s)$ are all continuous, and such that $\int_{a}^{b}\{K(s, t)\}^{2} d t$ is a limited function of $s$, for all values of $s$ in $(a, b)$, and if it be continuous in a closed connex set $G$, consisting of a domain and its boundary, and if the series $\sum_{n=1} \frac{\phi_{n}(s) \phi_{n}(t)}{\lambda_{n}}$ is uniformly convergent in $G$, then it converges throughout $G$ to the sum $K(s, t)$.

For the series converges in $G$ to a function that is continuous in $G$. Moreover, $G$ has its measure greater than zero, and thus, in the arbitrary small neighbourhood of any point of $G$, there are points which do not belong to the exceptional set. Hence $K(s, t)$ cannot differ at any point of $G$ from that funetion, continuous in $G$, which represents the sum of the series.

For example, if $K(s, t)$ is continuous except when $s=t$, where it may have any finite or infinite discontinuities consistent with the condition that $\int_{a}^{b}\{K(s, t)\}^{2} d t$ should exist for each value of $s$ as a limited function of $s$, and if $\sum_{n=1} \frac{\phi_{n}(s) \phi_{n}(t)}{\lambda_{n}}$ is uniformly convergent for all values of $s$ and $t$, such that $|s-t| \geqslant e$, when $\epsilon$ is an arbitrary positive number, then the series $\sum_{n=1} \frac{\phi_{n}(s) \phi_{n}(t)}{\lambda_{n}}$ converges to $K(s, t)$ at all points such that $s \neq t$. This applies, for example, to the case $K(s, t)=\frac{f(s, t)}{|s-t|^{\alpha}}$, where $f(s, t)$ is a continuous function, and $a<\frac{1}{2}$.

5. Let all the numbers $\lambda_{1}, \lambda_{2}, \ldots$, be positive, and let $K(s, t)$ be continuous ; then, in accordance with a known theorem due to Hilbert,*

* See E. Schmidt's paper in the Math. Amnalen, Vol. 63, p. 453. 
we have

$$
\int_{a}^{b} \int_{a}^{b} K(s, t) f(s) f(t) d s d t=\Sigma \frac{1}{\lambda_{n}}\left\{\int_{a}^{l} f(s) \phi_{n}(s) d s\right\}^{2},
$$

where $f(s)$ is continuous in $(a, b)$. If $f(s)$ is chosen so as to vanish except in the interval $\left(s_{1}, s_{1}+e\right)$, and to be positive within that interval, the number $\epsilon$ being so chosen that $K(s, t)$ has the same sign as $K\left(s_{1}, s_{1}\right)$ in the square $s_{1} \leqslant s \leqslant s_{1}+\epsilon, s_{1} \leqslant t \leqslant s_{1}+\epsilon$, unless $K\left(s_{1}, s_{1}\right)=0$. It is clear that that sign must be positive, since the right-hand side of the above equation is positive ; bence $K\left(s_{1}, s_{1}\right) \geqslant 0$, and, as $s_{1}$ is any point, we have $K(s, s) \geqslant 0$. If we now consider the nucleus

$$
K(s, t)-\sum_{n=1}^{n=m} \frac{\phi_{n}(s) \phi_{n}(t)}{\lambda_{n}}
$$

which is symmetrical and continuous, and has only the positive characteristic numbers $\lambda_{n+1}, \lambda_{n+2}, \ldots$, ve see that

$$
K(s, s)-\sum_{n=1}^{n=m} \frac{\left\{\phi_{n}(s)\right\}^{2}}{\lambda_{n}} \geqslant 0 .
$$

Thus the series $\sum_{n=1} \frac{\left\{\phi_{n}(s)\right\}^{2}}{\lambda_{n}}$ is convergent for every value of $s$, and its sum is limited for all values of $s$.

It follows from theorem III that there exists a nucleus $k(s, t)$ such that

$$
\int_{a}^{b}\{k(s, t)\}^{2} d t=\sum_{n=1} \frac{\left\{\phi_{n}(s)\right\}^{2}}{\lambda_{n}},
$$

of which the characteristic numbers are $\lambda_{1}^{\frac{1}{2}}, \lambda_{2}^{\frac{1}{2}}, \ldots$, and of which $\phi_{1}(s), \phi_{2}(s), \ldots$ are the characteristic functions. This nucleus is unique, except for equivalent ones.

The repeated nucleus

$$
k_{2}(s, t)=\int_{a}^{b} k\left(s, t^{\prime}\right) k\left(t, t^{\prime}\right) d t^{\prime}
$$

has for its sole characteristic values $\lambda_{1}, \lambda_{2}, \ldots$, and for its sole characteristic functions $\phi_{1}(s), \phi_{2}(s), \ldots$ (see $\S 1$ ).

Hence, since $\int_{a}^{b}\left\{k_{2}(s, t)\right\}^{2} d t$ is a limited function of $s$, it must, in accordance with what has been shewn in theorem III, be equivalent to $K(s, t)$, and therefore by the theorem I of $\S 2$, we see that $\sum_{n=1} \frac{\phi_{n}(s) \phi_{n}(t)}{\lambda_{n}}$ converges uniformly to $K(s, t)$. 
If a finite number of the numbers $\left\{\lambda_{n}\right\}$ be negative, we may apply the above result to the nuclens

$$
K(s, t)-\sum_{n=1}^{n=r} \frac{\phi_{n}(s) \phi_{n}(t)}{\lambda_{n}},
$$

where $r$ is so large that all the negative characteristic numbers are included in the first $r$. This nucleus has its characteristic constants $\lambda_{r+1}, \lambda_{r+2}, \ldots$ all positive, and the above result may be applied to it. It has thus been shewn that-

VII. If the symmetrical nucleus $K(s, t)$ be continuous, and all its eharacteristic constants, with the possible exception of a finite number of them, are of one sign, then $\sum_{n=1} \frac{\phi_{n}(s) \phi_{n}(t)}{\lambda_{n}}$ converges uniformly to $K(s, t)$ as sum.

This is Mercer's theorem.*

6. The theorem of Hilbert quoted in $\$ 5$ will now be extended to the case of discontinuous functions of which the squares are summable.

We shall assume that $K(s, t)$ is limited only by the condition that $\int_{a}^{b}\{K(s, t)\}^{2} d t$ exists for each value of $s$, and is a limited function of $s$ in the interval $(a, b)$. Let $p(t)$ be a function whose square is summable in the interval $(a, b)$. Then, if $g(s)=\int_{a}^{b} K(s, t) p(t) d t$, we see that $g(s)$ is a limited summable function.

Consider the function

$$
F(s)=g(s)-\sum_{n=1}^{\infty} \phi_{n}(s) \int_{a}^{b} g(t) \phi_{n}(t) d t .
$$

The series in the second term on the right-hand side is equivalent to

$$
\sum_{n=1}^{\infty} \lambda_{n} \int_{a}^{b} K(s, t) \phi_{n}(t) d t \int_{a}^{b} \int_{a}^{b} K\left(t, t^{\prime}\right) p\left(t^{\prime}\right) \phi_{n}(t) d t d t^{\prime},
$$

or to $\quad \sum_{n=1}^{\infty} \int_{a}^{b} K(s, t) \phi_{n}(t) d t \int_{a}^{b} \phi_{n}\left(t^{\prime}\right) p\left(t^{\prime}\right) d t^{\prime}$,

* See Phil. Trans., Vol. 209A. Another proof has been given by Kneser, in the Rend. di Palermo, Vol. xxxvir, p. 195. 
which, in accordance with a theorem * due to E. Schmidt, is absolutely and uniformly convergent.

We have now

$$
\int_{a}^{b} F(s) \phi_{n}(s) d s=\int_{a}^{b} g(s) \phi_{n}(s) d s-\int_{a}^{b} g(t) \phi_{n}(t) d t=0,
$$

and hence, in accordance with what has been shewn in $\S 9$, we see that

$$
\int_{a}^{b} K(s, t) F(t) d t=0 .
$$

We have now

$$
\int_{a}^{b}\{F(s)\}^{2} d s=\int_{a}^{b} F(s) g(s) d s=\int_{a}^{b} \int_{a}^{b} F(s) K(s, t) p(t) d s d t=0 .
$$

It follows that $F(s)=0$ for every value of $s$, with the possible exception of those belonging to a set of measure zero.

Therefore the uniformly convergent series

$$
\sum_{n=1} \phi_{n}(s) \int_{a}^{b} g(t) \phi_{n}(t) d t
$$

converges to $g(s)$ for all values of $s$ with the possible exception.

The series is equivalent to

$$
\sum_{n=1} \frac{\phi_{n}(s)}{\lambda_{n}} \int_{a}^{b} \phi_{n}(t) p(t) d t
$$

Multiplying by $q(s) d s$ and integrating, we have

$$
\sum_{n=1}^{\infty} \frac{1}{\lambda_{n}} \int_{a}^{b} \phi_{n}(s) q(s) d s \int_{a}^{b} \phi_{n}(t) p(t) d t=\int_{a}^{b} \int_{a}^{b} K(s, t) p(t) q(s) d s d t,
$$

where $q(s)$ is a function whose square is summable: The legitimacy of the term-by-term integration is unaffected by the possible existence of the exceptional set of points of measure zero.

We have now shewn that

$$
\int_{a}^{b} \int_{a}^{b} K(s, t) p(t) q(s) d s d t=\sum_{n=1}^{\infty} \frac{1}{\lambda_{n}} \int_{a}^{b} \phi_{n}(s) q(s) d s \int_{a}^{b} \phi_{n}(s) p(s) d s,
$$

where $p(s), q(s)$ are any functions whose squares are summable in $(a, b)$, 
and where. $K(s, t)$ is subject only to the condition that $\int_{a}^{b}\{K(s, t)\}^{2} d t$ exists for each value of $s$ and is limited as a function of $s$.

Let $p(s)$ and $q(s)$ be identical ; the theorem then becomes

$$
\int_{a}^{b} \int_{a}^{b} K(s, t) p(s) p(t) d s d t=\sum_{n=1}^{\infty} \frac{1}{\lambda_{n}}\left\{\int_{n}^{b} \phi_{n}(s) p(s) d s\right\}^{2} .
$$

7. Let it now be assumed that $K(s, t)$ is such that all the characteristic numbers $\left\{\lambda_{n}\right\}$ are positive. Let $p(s)$ be defined by $p(s)=1$, when $s_{1}-\epsilon \leqslant s \leqslant s_{1}+\epsilon$, and $p(s)=0$, for all other values of $s$, where $\epsilon$ is a fixed positive number, and $s_{1}$ a fixed value of $s$.

We see then that

$$
\iint K(s, t) d s d t
$$

taken over a square with its centre at the point $\left(s_{1}, s_{1}\right)$ and its sides of length $2 \epsilon$ parallel to the axes, is essentially positive.

Let us now assume that all the characteristic functions $\left\{\phi_{n}(s)\right\}$ are continuous, and let us apply the result just obtained to the nucleus

$$
K(s, t)-\sum_{n=1}^{n=n} \frac{\phi_{n}(s) \phi_{n}(t)}{\lambda_{n}},
$$

which is such that $\int_{a}^{b}\left\{K(s, t)-\sum_{n=1}^{n=n} \frac{\phi_{n}(s) \phi_{n}(t)}{\lambda_{n}}\right\}^{2} d t$,

or

$$
\int_{n}^{b}\{K(s, t)\}^{2} d t-\sum_{n=1}^{m}\left\{\frac{\phi_{n}(s)}{\lambda_{n}}\right\}^{2},
$$

is a limited function of $s$, the value of $m$ being fixed.

We see that

$$
\frac{1}{4 \epsilon^{2}} \iint K(s, t) d s d t-\frac{1}{4 \varepsilon^{2}} \iint_{n=1}^{n=m} \frac{\phi_{n}(s) \phi_{n}(t)}{\lambda_{n}} d s d t
$$

is essentially positive for each value of $m$ and each value of $\epsilon$; the integrals being taken over the square with centre at $\left(s_{1}, s_{1}\right)$.

Now $\sum_{n=1}^{n=m} \frac{\phi_{n}(s) \phi_{n}(t)}{\lambda_{n}}$ being a continuous function of $(s, t)$, we see that.

$$
\frac{1}{4 \epsilon^{2}} \iint \sum_{n=1}^{n=m} \frac{\phi_{n}(s) \phi_{n}(t)}{\lambda_{i n}} d s d t=\sum_{n=1}^{n=m} \frac{\left\{\phi_{n}\left(s_{1}\right)\right\}^{2}}{\lambda_{n}}+\eta_{m},
$$

where $\eta_{m}$ converges to zero as $\epsilon$ does so, and thus can be made arbitrarily small by taking $e$ small enough, for each fixed value of $m$. 
Thus $\quad \frac{1}{4 \epsilon^{2}} \iint K(s, t) d s d t-\sum_{n=1}^{n=m} \frac{\left\{\phi_{n}\left(s_{1}\right)\right\}^{2}}{\lambda_{n}}-n_{m}$ is essentially positive.

If $K(s, t)$ be such that it is not infinitely discontinnous at the point $\left(s_{1}, s_{1}\right)$, we can choose $\epsilon$ so small that $|K(s, t)|$ is limited in the square $s_{1}-\epsilon \leqslant s \leqslant s_{1}+\epsilon, s_{1}-\epsilon \leqslant t \leqslant s_{1}+\epsilon$.

In that case

$$
\frac{1}{4 \epsilon^{2}} \iint K(s, t) d s d t \leqslant N,
$$

where $N$ denotes the upper limit of $|K(s, t)|$ in the square so chosen. We now see that the series $\sum_{n=1} \frac{\left\{\phi_{n}\left(s_{1}\right)\right\}^{2}}{\lambda_{n}}$ cannot diverge, for if it diverges we can choose $m$ so large that

$$
\sum_{n=1}^{n=m} \frac{\left\{\phi_{n}\left(s_{1}\right)\right\}^{2}}{\lambda_{n}}>N
$$

and since $\eta_{n}$ may for this value of $m$ be taken arbitrarily small by proper choice of $\epsilon$, the expression

$$
\frac{1}{4 \epsilon^{2}} \iint K(s, t) d s d t-\sum_{n=1}^{n=m} \frac{\left\{\phi_{n}(s)\right\}^{2}}{\lambda_{n}}-\eta_{m}
$$

would then be negative, which is impossible.

Therefore the series $\sum_{n=1} \frac{\left\{\phi_{n}\left(s_{1}\right)\right\}^{2}}{\lambda_{n}}$ is convergent, provided $K(s, t)$ is not infinitely discontinuous at the point $\left(s_{1}, s_{1}\right)$.

In case $\sum_{n=1} \frac{\left\{\phi_{n}\left(s_{1}\right)\right\}^{2}}{\lambda_{n}}$ is divergent, $K(s, t)$ must be infinitely discontinuous at $\left(s_{1}, s_{1}\right)$.

In case $K(s, t)$ is such as to have no infinite discontinuity on the straight line $s=t$, the series $\Sigma \frac{\left\{\phi_{n}(s)\right\}^{2}}{\lambda_{n}}$ is convergent for every value of $s$ in $(a, b)$. It then follows that the series $\sum_{n=1} \frac{\phi_{n}(s) \phi_{n}(t)}{\lambda_{n}}$ is everywhere absolutely convergent.

Employing the theorem $V$ established in $\$ 4$, we now have the following theorem :-

VIII. If $K(s, t)$ be any symmetrical nucleus for which all the characteristic numbers are positive and the characteristic functions all continuous, such that $\int_{a}^{b}\{K(s, t)\}^{2} d t$ is a limited function of $s$, and also such 
as not to be infinitely discontinuous at any point on the straight line $s=t$, the series $\Sigma \frac{\phi_{n}(s) \phi_{n}(t)}{\lambda_{n}}$ converges to $K(s, t)$ everywhere with the possible exception of points of a set of which the sections by lines parallel to the axes have linear measure zero.

This is an extension of Mercer's theorem to the case of discontinuous nuclei with positive characteristic numbers. It is easily seen that it can be extended to the case in which a finite set of the characteristic numbers are negative.

8. Let $K(s, t)$ be any symmetrical nucleus for which $\left\{\phi_{n}(s)\right\}$ and $\left\{\lambda_{n}\right\}$ are the characteristic functions and constants, and - let it be assumed that the series $\sum_{n=1} \frac{1}{\left|\lambda_{n}\right|}$ is convergent.

Let $\epsilon_{1}, \epsilon_{9}, \ldots$, denote a sequence of decreasing positive numbers such that $\epsilon_{1}+\epsilon_{2}+\ldots$ converges to a positive number $\xi$ arbitrarily chosen. Let $m_{1}, m_{2}, m_{3}, \ldots$, be a sequence of increasing integers so chosen that

$$
\sum_{m_{1}}^{\infty} \frac{1}{\left|\lambda_{n}\right|}<\epsilon_{1}^{2}, \quad \sum_{m_{2}}^{\infty} \frac{1}{\left|\lambda_{n}\right|}<\epsilon_{2}^{2}, \ldots
$$

We have then

$$
\begin{aligned}
& \int_{a}^{b} \sum_{m_{1}}^{m_{3}-1} \frac{\left\{\phi_{n}(s)\right\}^{2}}{\left|\lambda_{n}\right|} d s<\epsilon_{1}^{2}, \\
& \int_{a}^{b} \sum_{m_{3}}^{m_{3}-1} \frac{\left\{\left.\phi_{n}(s)\right|^{2}\right.}{\left|\lambda_{n}\right|} d s<\mathrm{\epsilon}_{2}^{2}, \ldots .
\end{aligned}
$$

It follows that

$$
\sum_{m_{1}}^{m_{2}-1} \frac{\left\{\phi_{n}(s)\right\}^{2}}{\left|\lambda_{n}\right|}<\epsilon_{1}
$$

in the points of a set of linear measure $>b-a-\epsilon_{1}$; that

$$
\sum_{m_{1}}^{m_{3}-1} \frac{\left\{\phi_{n}(s)\right\}^{2}}{\left|\lambda_{n}\right|}<\epsilon_{2}
$$

in the points of a set of linear measure $>b-a-\epsilon_{2}$, and so on.

It follows that in the points of a set of linear measure $>b-a-\zeta$,

for all values of $r$.

$$
\sum_{m_{1}}^{m_{1}+r} \frac{\left\{\phi_{n}(s)\right\}^{2}}{\left|\lambda_{n}\right|}<\xi
$$

Now choose a set of values of $\xi$, say $\xi_{1}, \xi_{2}, \ldots$ converging to zero, and such that $\xi_{1}+\xi_{2}+\ldots$ converges to a positive number $\delta$ arbitrarily small. 
We see then that in a set of measure $>b-a-\delta$, the series $\sum_{n=1} \frac{\left\{\phi_{n}(s)\right\}^{2}}{\left|\lambda_{n}\right|}$ converges uniformly. Since $\delta$ is arbitrarily small, we see that $\sum \frac{\left\{\phi_{n}(s)\right\}^{2}}{\lambda_{n}}$ is convergent for all values of $s$ that do not belong to some set of linear messure zero.

Since

$$
\left|\frac{\phi_{n}(s) \phi_{n}(t)}{\lambda_{n}}\right| \leqslant \frac{1}{2} \frac{\left\{\phi_{n}(s)\right\}^{2}}{\mid \lambda_{n} !}+\frac{1}{2} \frac{\left\{\phi_{n}(t)\right\}^{2}}{\left|\lambda_{n}\right|}
$$

we see that the series $\Sigma \frac{\phi_{n}(s) \phi_{n}(t)}{\lambda_{n}}$ is absolutely convergent, provided neither $s$ nor $t$ belong to the exceptional linear set. We thus see that $\sum_{n=1} \frac{\phi_{n}(s) \phi_{n}(t)}{\lambda_{n}}$ is convergent, except possibly at points of a set of which the plane measure is zero.

It has thus been shewn that:-

IX. If $K(s, t)$ be any symmetrical nucleus not necessarily continuous or limited, such that the series of which the terms are the reciprocals of its characteristic numbers is absolutely convergent, then $\sum_{n=1} \frac{\phi_{n}(s) \phi_{n}(t)}{\lambda_{n}}$ is absolutely convergent, except possibly at points of a set of which the plane measure is zero.

Employing theorem V, we now obtain the following theorem :-

$\mathrm{X}$. If $K(s, t)$ be any symmetrical nucleus, limited or unlimited, such that its characteristic functions are continuous, and such that $\int_{a}^{b}\left\{K(s, t)^{2} d t\right.$ is a limited function of $s$, then, if the series of which the terms are the reciprocals of the characteristic numbers is absolutely convergent, the series $\sum_{n=1} \frac{\phi_{n}(s) \phi_{n}(t)}{\lambda_{i n}}$ converges to $K(s, t)$ with the possible exception of points $(s, t)$ of a set of which the plane measure is zero.

9. Let $K(s, t)$ be such that, provided $|s-t|$ is $\geqslant \epsilon$, where $\epsilon$ is an arbitrarily chosen positive number, $\left|\sum_{n=1}^{n=m} \phi_{n}(s) \phi_{n}(t)\right|$ is less than some fixed positive number, for all values of $m$, and for all the values of. $s$ and $t$ which satisfy the prescribed condition. Also let us assume that all the characteristic numbers $\left\{\lambda_{n}\right\}$ corresponding to $K(s, t)$ are positive. 
It can then be shewn, by employing Abel's lemma, that the series $\sum_{n=1} \frac{\phi_{n}(s) \phi_{n}(t)}{\lambda_{n}}$ converges uniformly for all the values of $s$ and $t$, such that $|s-t| \geqslant \varepsilon$.

For

$$
\begin{aligned}
\sum_{n=m+1}^{n=m+p} \frac{\phi_{n}(s) \phi_{n}(t)}{\lambda_{n}}=\frac{1}{\lambda_{m+p+1}} & \sum_{m+1}^{m+p} \phi_{n}(s) \phi_{n}(t) \\
& +\sum_{q=1}^{q=p}\left[\left(\frac{1}{\lambda_{m+q}}-\frac{1}{\lambda_{m+p+1}}\right) \sum_{m+1}^{m+q} \phi_{n}(s) \phi_{n}(t)\right] ;
\end{aligned}
$$

and since

$$
\frac{1}{\lambda_{m+q}}-\frac{1}{\lambda_{m+p+1}} \geqslant 0
$$

We have $\left|\sum_{n=m+1}^{n=m+p} \frac{\phi_{n}(s) \phi_{n}(t)}{\lambda_{n}}\right|<\frac{P}{\lambda_{m+p+1}}+P\left(\frac{1}{\lambda_{m+1}}-\frac{1}{\lambda_{m+p+1}}\right)<\frac{P}{\lambda_{m+1}}$,

where $P$ is a fixed positive number, dependent only on $\epsilon$. Since $\frac{1}{\lambda_{m+1}}$ is arbitrarily small, by making $m$ large enough, the uniform convergence of the series is established.

If we assume that $K(s, t)$ is continuous except on the line $s=t$, where it is infinitely discontinuous, and that $\int_{a}^{b}\{K(s, t)\}^{2} d t$ is a limited function of $s$, the functions $\left\{\phi_{n}(s)\right\}$ are all continuous, and the theorem VI of $\S 4$ is applicable.

We obtain then the following theorem :-

XI. If $K(s, t)$ is continuous except on the line $s=t$, where it may be infinitely discontinuous, but such that $\int_{a}^{b}\{K(s, t)\}^{2} d t$ is a limited function of $s$, and if $\left|\sum_{n=1}^{n=m} \phi_{n}(s) \phi_{n}(t)\right|$ is less than some fixed finite number, independent of $m$, for all values of $s$ and $t$ such that $|s-t| \geqslant \epsilon$, where $\epsilon$ is an arbitrarily chosen positive number, then the series $\sum_{n=1} \frac{\phi_{n}(s) \phi_{n}(t)}{\lambda_{n}}$ converges uniformly to $K(s, t)$ for all values of $s$ and $t$ such that

$$
|s-t| \geqslant \epsilon \text {. }
$$

This theorem is applicable to the case of the Sturm-Liouville functions and to other orthogonal functions which arise in connexion with linear differential equations. 
10. Let $K(s, t)$ be such that $\int_{a}^{b}\{K(s, t)\}^{2} d t$ is a limited function of $s$, and that the repeated function of $K(s, t)$ is equivalent to a continuous function $K_{2}(s, t)$.

Then, as has been shewn in theoren I, $\sum_{n=1} \frac{\phi_{n}(s) \phi_{n}(t)}{\lambda_{n}}$ converges uniformly to $K_{2}(s, t)$.

Therefore $\Sigma\left(\frac{\phi_{n}(s)}{\lambda_{n}}\right\}^{2}$ converges to a continuous function of $s$.

Let us consider separately those values of $\lambda$ which are positive and those which are negative; let the former be denoted by

and the latter by

$$
\begin{aligned}
& \lambda_{1}^{(P)}, \lambda_{2}^{(P)}, \lambda_{3}^{(P)}, \ldots, \\
& \lambda_{1}^{(N)}, \lambda_{2}^{(N)}, \lambda_{3}^{(N)}, \ldots,
\end{aligned}
$$

each series being arranged in order of increasing absolute magnitude. It is then elear that the two series

$$
\sum_{n=1}\left\{\frac{\phi_{n}^{(P)}(s)}{\lambda_{n}^{(P)}}\right\}^{2}, \quad \sum_{n=1}\left\{\frac{\phi_{n}^{(N)}(s)}{\lambda_{n}^{(N)}}\right\}^{2}
$$

are each convergent and have limited sum functions.

Let $K^{(1)}(s, t)$ be that nucleus, constructed as in $\S 3$, which has $\left\{\phi_{n}^{(P)}(s)\right\},\left\{\lambda_{n}^{(P)}\right\}$ for its sole characteristic functions and numbers, and let $K^{(2)}(s, t)$ be the nucleus, similarly constructed, which has $\left\{\phi_{n}^{(N)}(s\}\right.$, $\left\{\lambda_{n}^{(N)}\right\}$ for its sole characteristic functions and numbers. We have then

$$
\int_{a}^{b}\left\{K^{(1)}(s, t)\right\}^{2} d t=\sum_{n=1}\left\{\frac{\phi_{n}^{(P)}(s)}{\lambda_{n}^{(P)}}\right\}^{2}, \quad \int_{a}^{b}\left\{K^{(2)}(s, t)\right\}^{2} d t=\sum_{n=1}\left\{\frac{\phi_{n}^{(N)}(s)}{\lambda_{n}^{(N)}}\right\}^{2} ;
$$

and therefore

$$
\int_{a}^{b}\left\{K^{(1)}(s, t)\right\}^{2} d t+\int_{a}^{b}\left\{K^{(2)}(s, t)\right\}^{2} d t=\int_{a}^{b}\{K(s, t)\}^{2} d t .
$$

Since $\phi_{n}^{(N)}(t)$ is orthogonal to all the characteristic functions of $K^{(1)}(s, t)$, we have

$$
\int_{a}^{\grave{b}} K^{(1)}(s, t) \phi_{n}^{(N)}(t) d t=0 ;
$$

and similarly, we have $\int_{a}^{b} K^{(2)}(s, t) \phi_{n}^{(P)}(t) d t=0$. 
It follows that

$$
\int_{a}^{b}\left\{K^{(2)}(s, t)+K^{(2)}(s, t)\right\} \phi_{n}^{(P)}(t) d t=\lambda_{n}^{(P)} \phi_{n}^{(P)}(s),
$$

and

$$
\int_{a}^{b}\left\{K^{(1)}(s, t)+K^{(2)}(s, t)\right\} \phi_{n}^{(N)}(t) d t=\lambda_{n}^{(N)} \phi_{n}^{(N)}(s) ;
$$

Hence the nucleus $K^{(1)}(s, t)+K^{(2)}(s, t)$ has the same characteristic functions and constants as $K(s, t)$. It will be shewn that it has no other characteristic functions than these.

Since $K^{(1)}\left(s, t^{\prime}\right)$ is orthogonal to $\phi_{1}^{(N)}\left(t^{\prime}\right), \phi_{2}^{(N)}\left(t^{\prime}\right), \ldots$, we have

$$
\int_{a}^{b} K^{(i)}\left(s, t^{\prime}\right) K^{(2)}\left(t, t^{\prime}\right) d t^{\prime}=0,
$$

$$
\text { hence } \begin{aligned}
\int_{a}^{b}\left[K^{(1)}\left(s, t^{\prime}\right)+\right. & \left.K^{(2)}\left(s, t^{\prime}\right)\right]\left[K^{(1)}\left(t^{\prime}, t\right)+K^{(2)}\left(t^{\prime}, t\right)\right] d t^{\prime} \\
& =\int_{a}^{b} K^{(1)}\left(s, t^{\prime}\right) K^{(1)}\left(t, t^{\prime}\right) d t^{\prime}+\int_{a}^{b} K^{(2)}\left(s, t^{\prime}\right) K^{(2)}\left(t^{\prime}, t\right) d t^{\prime},
\end{aligned}
$$

and thus the repeated function of $K^{(1)}(s, t)+K^{(2)}(s, t)$ is the sum of the repeated functions of $K^{(1)}(s, t)$ and $K^{(2)}(s, t)$.

Also

$$
\int_{a}^{b}\{K(s, t)\}^{2} d t=\int_{a}^{b}\left\{K^{(1)}(s, t)+K^{(2)}(s, t)\right\}^{2} d t,
$$

since

$$
\int_{a}^{b} K^{(1)}(s, t) K^{(2)}(s, t) d t=0 .
$$

Since $\int_{n}^{b}\left\{K^{(1)}(s, t)+K^{(2)}(s, t)\right\}^{2} d t=\sum_{n=1}\left\{\frac{\phi_{n}^{(P)}(s)}{\lambda_{n}^{(P)}}\right\}^{2}+\sum_{n=1}\left\{\frac{\phi_{n}^{(N)}(s)}{\lambda_{n}^{(N)}}\right\}^{2}$,

it now follows that $K^{(1)}(s, t)+K^{(2)}(s, t)$ has no characteristic functions and constants other than those of $K(s, t)$.

The following result bas been established :-

XII. If $K(s, t)$ be such that its repeated function is equivalent to a continuous function, then $K(s, t)$ may be expressed as the sum of two functions $K^{(1)}(s, t), K^{(2)}(s, t)$, orthogonal to one another, and such that $K^{(1)}(s, t)$ has for its sole characteristic numbers those numbers corresponding to $K(s, t)$ which are positive; and such that $K^{(2)}(s, t)$ has for its sole characteristic numbers those numbers corresponding to $K(s, t)$ which are negative. The characteristic function corresponding to a characteristic number of $K^{(1)}(s, t)$ or of $K^{(2)}(s, t)$ is the same as the characteristic function of $K(s, t)$ corresponding to the same characteristic number. 
This theorem is applicable in particular to a continuous nucleus $K(s, t)$, which may have an infinite number of characteristic numbers of each sign.

If it were certain that $K^{(1)}(s, t), K^{(2)}(s, t)$ were not infinitely discontinuous on the straight line $s=t$, theorem VIII would be applicable to these nuclei, and the uniform convergence of $\Sigma \frac{\phi_{n}(s) \phi_{n}(t)}{\lambda_{n}}$ to $K(s, t)$ could be deduced. As, however, this possibility cannot be excluded, at all events a priori, no further general result has been obtained for the case of a continuous nucleus with an infinite number of characteristic numbers of each sign beyond that obtained in $\S 3$; that, for each point $(s, t)$, with the possible exception of points belonging to a set of measure zero, a sequence of values of $m$ can be so determined that $\sum_{n=1}^{n=m} \frac{\phi_{n}(s) \phi_{n}(t)}{\lambda_{n}}$ converges to $K(s, t)$ as $m$ goes through the values in the sequence. 